Volume 8, No.2, March - April 2019

International Journal of Advanced Trends in Computer Science and Engineering

Available Online at http://www.warse.org/IJATCSE/static/pdf/file/ijatcse23822019.pdf

https://doi.org/10.30534/ijatcse/2019/23822019

\title{
Development and Characterization of a Dimmable LED Luminaire for Body Detection, Tracking and Recognition under Natural and Artificial Low-light Illumination
}

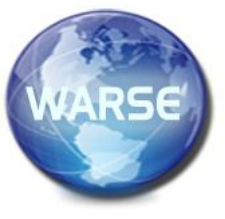

\begin{abstract}
Illumination of the scene is crucial in order the detection algorithm to carry out the computation task correctly. In this study, an LED based luminaire is developed for a computer vision system for the detection and tracking of pedestrian or human body in a video under different illumination. The illumination is provided using an LED lighting system where the intensity of illumination can be varied by changing the duty cycle of the pulse activating the LEDs. The mean of ambient light intensities captured by the image sensor is captured under different lighting conditions which will be useful in designing a control system that consider the amount of ambient lighting to set the setting of LED lighting activation. This adaptive system is useful to provide illumination that take into consideration ambient light intensities.
\end{abstract}

Key words : LED Lumainaire, Camera-based Illumination, Object Detection, Embedded System.

\section{INTRODUCTION}

Due to the increasing demand for energy and concerns for environmental pollution and degradation due to carbon emissions as a result of human activities, many research activities were focused in generating technologies to optimize and conserve the use of energy. One of such technologies is lighting technologies. Recent developments in semiconductor paved the way in producing Light Emitting Diodes (LED) that can be used in lighting because of it characteristics that lend to lower energy consumption, longer operating life among others.

\section{RELATED WORKS}

LED is a semiconductor device that emits light at a certain wavelength, much different from conventional light sources such as incandescent, fluorescent, and gas-discharge lamps. It has no filament or gas-filled glass bulb that can break. In the EU, incandescent bulbs were already banned for use. In the photometric study of LED light sources [1] to determine the suitability of LED replacement to conventional light source, the luminous intensity from a LED light source is calculated using Eq. 1 which provides the measure of total light within the range of the human visual response. A measurement error of less than $1 \%$ is obtained if measurement is taken from a light source which is considered as a point source. According to the author, a light source is considered as a point source "if the photometric distance is 5 times longer than the longest dimension of the light active surface."

$$
I=E x r^{2}
$$

where:

$\mathrm{I}$ is the luminous intensity (cd)

$\mathrm{E}$ is the illuminance (lx)

$\mathrm{R}$ is the photometric distance $(\mathrm{m})$.

In [2], an evaluation of LED luminaires was conducted to determine if there are direct substitute for traditional incandescent light bulbs based on the basic evaluation criterion of illuminance, uniformity and glare limitation in the place of work. Their study indicated that not all light sources can be considered substitutes of traditional light bulbs. Notable to their study is the use of the testbed in conducting the photometric study. The effect of heat generated in the luminaire greatly affects the performance of luminaires using LED lighting technology. [3] in 2016 presented the design of heat sink to dissipate junction temperatures of LED to maintain good performance of LED luminaires. For luminaires operating at continuous or very long operating hours, junction temperature generated in the LED lighting must be removed effectively.

The Photometric Analysis of LED light source helped in establishing the operating characteristics of the LED luminaire that will be used in this study. Establishing the beam pattern generated by the LED luminaire provided the insight in designing and implementing the LED lighting subsystem based on the apex angle of the LEDs to be used in this research. Since it was also established that there are no direct replacement to any luminaire, it is necessary that the characteristics of the lighting subsystem has to be characterized in terms of of luminous intensity and beam pattern.

\section{DESIGN CONSIDERATIONS}

\section{A. Lighting Subsystem}

The LED Lighting Subsystem is composed of three parts namely, the LED luminaire, driver circuit and the PWM controller. The block diagram is shown in Figure 1. 


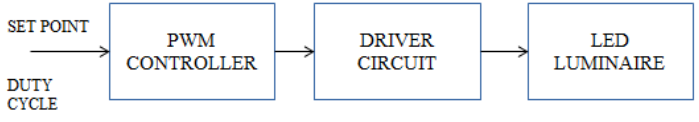

Figure 1: Block diagram of the LED lighting system

The LED luminaire is implemented using $1610 \mathrm{~mm}$ super-bright white light LEDs. Each LED is connected in series with a current limiting resistor with a resistance value chosen to provide a forward current flow to IF $=20 \mathrm{~mA}$ with a forward voltage $\mathrm{VF}=3 \mathrm{~V}$. At this operating point, the LED will emit 1300 mcd of whit light. Sixteen of these strings are connected in parallel for a total current of 320 $\mathrm{mA}$. The LEDs are mounted on two PCB boards, with each board having eight LEDs each. A TIP 29 power transistor is used as switching element. The required base current of the TIP transistor is provided by a 2 N2219 transistor connected to the former transistor in a darlington configuration. PWM controller implementation is through the use of an Arduino Uno microcontroller. Arduino has a built in PWM pin and a built in library for PWM applications that can be easily implemented. In this implementation, pin 6 is used PWM as the output pin for PWM generation. The LED lighting assembly is shown in Figure 2.

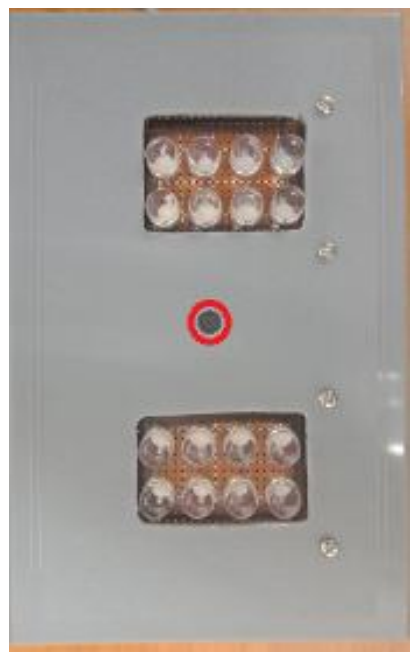

Figure 2: LED lighting assembly used in this study with image sensor mounted (enclosed with red circle)

\section{B. Implementing the PWM Lighting Controller}

The PWM controller is implemented on an Arduino Uno board. A 5-bit value encodes the PWM duty cycle: $0 \%$ $100 \%$ with $5 \%$ increments is applied on 5 GPIO pins of the Arduino board. PWM pin 6 drives the transistor driver circuit activating the intensity of the LEDs. The PWM duty cycle is supplied to the Arduino board through a control panel composed of push button switches. The system is amenable to interfacing to an external embedded system such as a Raspberry Pi. Such arrangement is shown Figure 3.

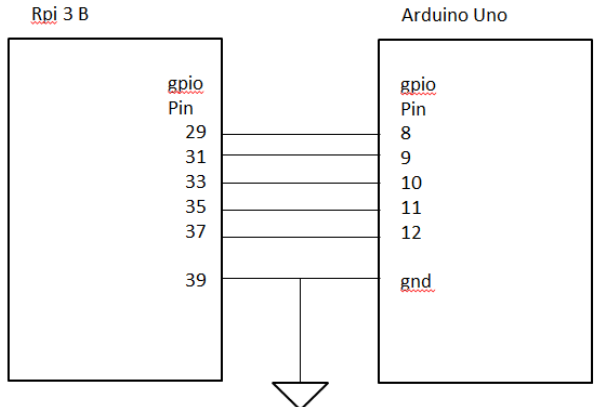

Figure 3: Raspberry Pi 3 B board - Arduino Uno board interfacing

\section{Characterizing the LED Lighting subsystem}

The characterization of the LED lighting system validates the light intensity output generated by the led luminaire. For each duty cycle from $5 \%$ to $100 \%$ with $5 \%$ increments, the lux level is measure using the Sekonic C700R Spectrometer. This experiment measures the illumination received by the object (human person) at different duty cycle of PWM, at distances of 20,18,16,14,12,10,8, 6, 4, and 2 feet from the light source. Measurements of the light intensity were taken based on the distance from the light source. The top view representation of the PATH assignment of the test bed showing where measurements were made is shown in Figure 4. DF is the distance between the measurement point and the light source. Measurements were taken in points along PATH 5.

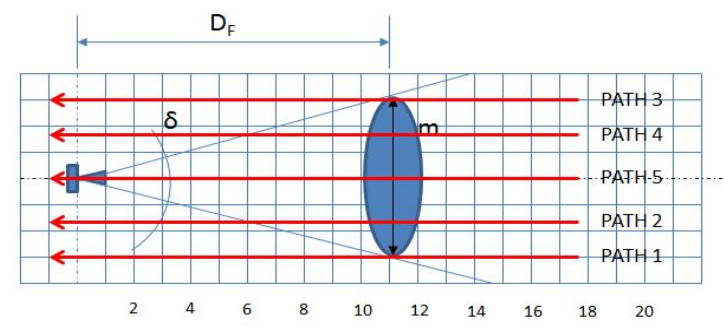

Figure 4: The top view representation of the PATH assignment of the test bed.

Another factor considered in the measurement is the elevation of the light source. In this work, the height of the LED lighting is 8 feet and depressed at an angle $\delta$ degrees. The setup is represented in Figure 5.

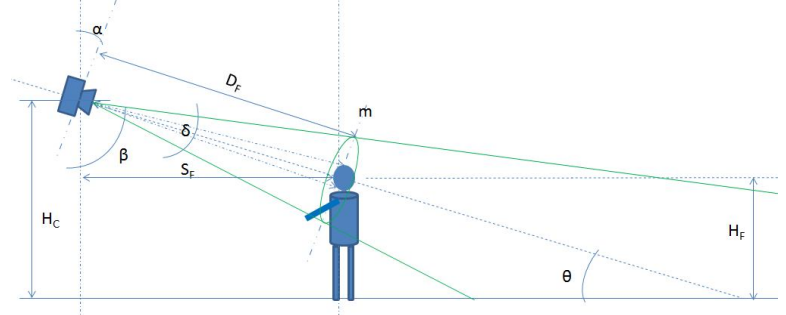

Figure 5: Side view representation of the test bed showing the light source and the beam pattern it produce. 
Using Figure 5 which shows the nomenclature of lighting in the test bed, the parameters that are involved in the manner the image sensor must be installed are indicated, based on the illumination configuration used in this research. In addition to $\delta$, is the spread angle of the beam, $m$ is the spread distance which is the diameter of the base of the cone, DF is the distance of the base of the cone from the image sensor defined earlier, other parameters will also affect the performance of the computer vision system. These include the height of the camera HF, the angle of depression $\beta$ and the horizontal distance of the detected object from the image sensor SF. The angle of elevation $\theta$, which is the complement of $\beta$, is the angle formed by the axis of the camera FOV with horizontal, will be used if the camera is installed behind the object.

\section{Measuring the light intensity received by the image sensor}

The illumination level received by the image sensor is characterized by taking the mean of pixel intensities in an image for different PWM duty cycle settings. The readings will provide the data as fuzzy input in order to activate the LED lighting system when the ambient light is sufficient for body and face detection as well as face recognition to be performed by the computer vision system correctly.

\section{RESULTS AND DISCUSSION}

The power drawn by the LED lighting system is measured in order to determine its power drain from the source. This is also necessary to calculate the efficiency of the lighting system. Table 1 summarized the current drain of the LED lighting. The lighting system is activated in increment of 5 $\%$ duty cycle and the current is measured. Based on this measurement, the power drained by the LED lighting system is computed by multiplying the current with the supply voltage, 5 volts in this prototype. It can be seen from the results that for each 5\% duty cycle step, the lighting system draws 11 milli amperes of current from the source.

The simplistic design of the box appears to be a square or rectangle which is very similar to the books causes the number and feature of key points to be similar. This is why we get a $0 \%$ accuracy rate in the books category. It is also true to some cases that the plastic bags or paper bag is shape similar to a box and output the box category.
Table 1: Power drawn by the LED lighting system

\begin{tabular}{|c|c|c|}
\hline Duty Cycle & $\begin{array}{c}\text { Current drawn, } \\
\text { mA }\end{array}$ & $\begin{array}{c}\text { Power Requirement } \\
\text { @5Vdc }\end{array}$ \\
\hline 0 & 0 & 0 \\
\hline 5 & 11.2 & 56 \\
\hline 10 & 25 & 125 \\
\hline 15 & 37.9 & 160 \\
\hline 20 & 48.4 & 242 \\
\hline 25 & 62.2 & 311 \\
\hline 30 & 75.1 & 375 \\
\hline 35 & 88.1 & 440.5 \\
\hline 40 & 99.5 & 497.5 \\
\hline 45 & 108 & 540 \\
\hline 50 & 120 & 600 \\
\hline 55 & 134 & 670 \\
\hline 60 & 145 & 730 \\
\hline 65 & 161 & 805 \\
\hline 70 & 173 & 865 \\
\hline 75 & 189 & 945 \\
\hline 80 & 197 & 985 \\
\hline 85 & 250 & 1250 \\
\hline 90 & 275 & 1375 \\
\hline 95 & 300 & 1500 \\
\hline 100 & 325 & 1625 \\
\hline
\end{tabular}

Table 2 lists the light intensity measured at different points in the testbed. The measurements indicate the light output generated by the LED lighting system at different distance from the source. The LED luminaire developed for the study has a total output $14.4 \mathrm{~lm}$ (lumens). This is based on the specification on the datasheet. Each of the 10mm LED has an output of $0.75 \mathrm{~lm}-0.91 \mathrm{~lm}$ with a luminous efficiency of 80 $-100 \mathrm{~lm} / \mathrm{W}$ where; $1 \mathrm{~W}=\mathrm{lm} /(\mathrm{lm} / \mathrm{W})$.

The watt output of one LED is therefore $0.75 / 80 \mathrm{~lm} / \mathrm{W}$ which is equal to $0.0094 \mathrm{~W}$. For 16 LEDs, this is equal to $0.15 \mathrm{~W}$. This is the energy consumed by the luminaire and converted into light with the luminous flux of 14.4 lumens. The Apex angle for the LED is 30 degrees. Assuming that the luminaire Apex angle for the combine LED remains the same, the intensity of the light on the plane normal to the axis of the light beam, can be determined at any distance.

The light beam can be thought of having a shape of a cone where the vertex is from the light source and the base of the cone is the plane normal to the beam axis. The area of distribution of light will be the area of the base of the cone which is dependent on the spread of the beam. Fig 6 shows the spreading of the beam which is outlined by the black circle drawn to encircle it. 


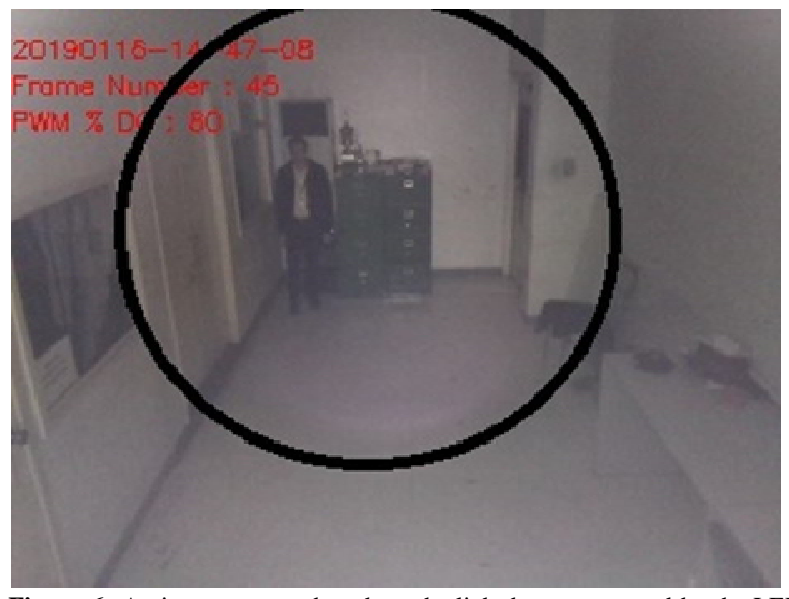

Figure 6: An image captured to show the light beam generated by the LED luminaire. A black circle is drawn to highlight the spreading of the beam.

Table 3 is a computation of the expected light intensity at different distances from the light source based on the output of the LEDs used in the design. Based on the measurement values, the entry for light intensities generated at 55\% duty cycle approximates the characteristics of the computed values. This provides qualitative assessment of the performance of the lighting system which indicates that the necessary illumination levels can be provided by the system. Measurement errors and the conditions by which these measurements were taken are factors that caused measurements and calculated values to vary. Most important is the LED device used in the luminaire where the exact specifications for the device are not readily available. Thus the performance of the lighting system is more on the qualitative aspects.

Table 3: Computed luminous intensity and the light spread index at different distances from the light source.

\begin{tabular}{|l|c|c|c|}
\hline & \multicolumn{3}{|l|}{ Luminous flux = 14.4 lm @ 30 degrees } \\
\hline $\begin{array}{l}\text { Distance, } \\
\text { feet }\end{array}$ & $\begin{array}{l}\text { Computed } \\
\text { Luminous } \\
\text { Intensity, lux }\end{array}$ & $\begin{array}{l}\text { Light } \\
\text { spread, } \\
\text { feet }\end{array}$ & $\begin{array}{l}\text { Coverage } \\
\text { area, sq. ft. }\end{array}$ \\
\hline 2 & 180.8 & 1.1 & 0.933 \\
\hline 4 & 45.19 & 2.14 & 3.62 \\
\hline 6 & 20.08 & 3.21 & 8.12 \\
\hline 8 & 11.3 & 4.3 & 14.42 \\
\hline 10 & 7.24 & 5.3 & 22.6 \\
\hline 12 & 5.02 & 6.4 & 32.5 \\
\hline 14 & 3.7 & 7.5 & 44.2 \\
\hline 16 & 2.82 & 8.6 & 57.8 \\
\hline 18 & 2.24 & 9.6 & 72.7 \\
\hline 20 & 1.81 & 10.7 & 90.3 \\
\hline
\end{tabular}

The intensity level of the light captured by the image sensor at different PWM duty cycle settings of the LED lighting is recorded in Table 4 alongside with the light intensities measured near the image sensor. A sample image that indicates the intensity level of the ambient lighting from a florescent lamp with only the background image capture is shown in Figure 7.

Table 4: Computed luminous intensity and the light spread index at different distances from the light source.

\begin{tabular}{|c|c|c|}
\hline Duty Cycle & $\begin{array}{l}\text { Image sensor, } \\
\text { mean } \\
\text { illumination }\end{array}$ & $\begin{array}{l}\text { Spectrophotometer } \\
\text { reading, lux }\end{array}$ \\
\hline 0 & 0 & Under \\
\hline 5 & 0 & Under \\
\hline 10 & 1 & Under \\
\hline 15 & 3 & Under \\
\hline 20 & 2 & Under \\
\hline 25 & 3 & 1.1 \\
\hline 30 & 4 & 1.2 \\
\hline 35 & 5 & 1.2 \\
\hline 40 & 7 & 1.2 \\
\hline 45 & 8 & 1.6 \\
\hline 50 & 10 & 1.5 \\
\hline 55 & 10 & 2 \\
\hline 60 & 11 & 2.2 \\
\hline 65 & 14 & 2.3 \\
\hline 70 & 14 & 3.6 \\
\hline 75 & 14 & 4.5 \\
\hline 80 & 17 & 5.8 \\
\hline
\end{tabular}

The lowest intensity of light that can be measure by the photometer is at 1.1 lux corresponding to a reading of 3 by the image sensor. This occurred at PWM duty cycle $25 \%$. Based on the values in Table IV, reading from $0-3$ represents DARK ambient lighting ( no lighting). Reading from 8 to 11 considered as BRIGHT, and 14 to 17 as VERY BRIGHT.

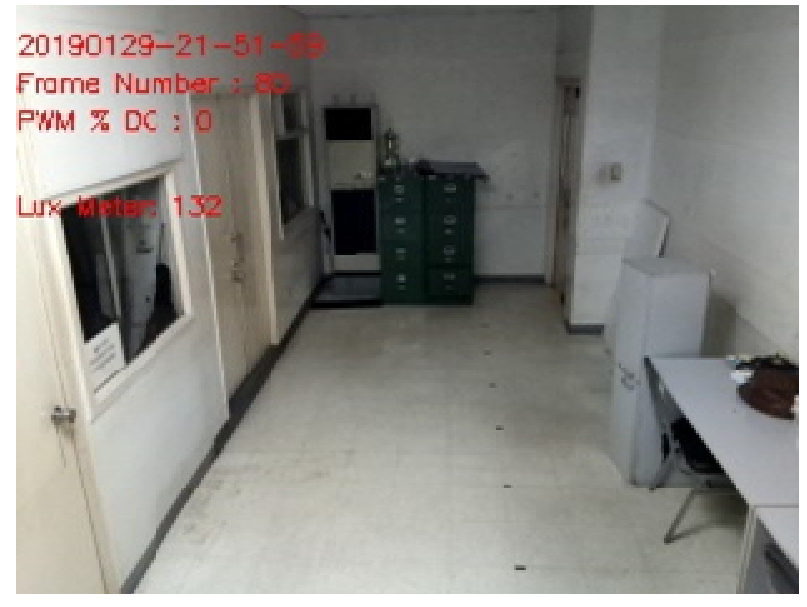

Figure 7: Image of the background of the testbed with the light intensity indicator (Lux Meter) reading 132.

As shown in Fig. 7, the Lux Meter indicates 132. This reading however is just a magnitude of the mean of pixel intensities of the image frame and is not the actual lux reading of the light intensity. 


\section{CONCLUSION}

In this section, the development of the LED lighting subsystem and the photometric analysis of the performance were described. Qualitative performance values obtained provide the basis for assessing the performance of the LED lighting system which makes the lighting subsystem capable of providing the illumination levels required by the computer vision subsystem to carry out detection, tracking and recognition of human object in an image. A way to determine the light intensity of the scene is also included in this work to provide a means of determining when to use the LED lighting system as a source of illumination. Measurement readings 3 and below indicate the absence of ambient or artificial lighting which requires the use of the LED lighting system as the source of illumination for the image sensor.

\section{REFERENCES}

[1] J. Škoda, P. Baxant, M. Krbal, S. Sumec, and T. Pavelka. "Photometry of LED sources". Przeglad Elektrotechniczny, R. 89 NR 6, 341 - 344, 2013. Retrieved from http://pe.org.pl/articles/2013/6/73.pdf.

[2] M. Zalesinska, S. Szwedek and A. Pawlak, "Evaluation of Lighting Parameters at the Workplace with the use Replacements for Incandescent Lamps", 2018 VII. Lighting Conference of the Visegrad Countries (Lumen V4), 2018.

https://doi.org/10.1109/LUMENV.2018.8521106

[3] A. Rozowicz, K. Baran and H. Wachta, "Radiation studies of the illumination lighting luminaires with LED technology", 2016 IEEE Lighting Conference of the Visegrad Countries (Lumen V4), 2016 https://doi.org/10.1109/LUMENV.2016.7745533

Table 2: Power drawn by the LED lighting system

\begin{tabular}{|c|c|c|c|c|c|c|c|c|c|c|}
\hline & \multicolumn{10}{|c|}{ Distance, feet } \\
\hline Duty cycle & 2 & 4 & 6 & 8 & 10 & 12 & 14 & 16 & 18 & 20 \\
\hline 5 & 15 & 8.6 & 3.7 & 2.1 & 1.2 & & & & & \\
\hline 10 & 30.7 & 15.3 & 7 & 4.2 & 2.5 & 1.9 & 1 & & & \\
\hline 15 & 47.8 & 23.3 & 10.9 & 6.3 & 3.9 & 2.8 & 1.9 & 1.6 & 1.1 & \\
\hline 20 & 62 & 30.5 & 14.4 & 8.3 & 5.1 & 3.8 & 2.6 & 2.1 & 1.5 & 1.5 \\
\hline 25 & 61.4 & 36.3 & 18 & 10.4 & 6.6 & 4.9 & 3.4 & 2.7 & 2.1 & 1.9 \\
\hline 30 & 73.3 & 44.8 & 22.1 & 12.7 & 8.1 & 5.7 & 4.1 & 3.4 & 2.6 & 2.3 \\
\hline 35 & 97 & 52.8 & 26.4 & 15.3 & 10.2 & 7.2 & 5.1 & 4 & 3 & 2.7 \\
\hline 40 & 94.4 & 58.7 & 25.7 & 17.3 & 11 & 7.9 & 5.8 & 4.6 & 3.6 & 3 \\
\hline 45 & 85.5 & 65.8 & 29.6 & 18.4 & 12.2 & 8.7 & 6.4 & 5.1 & 3.9 & 3.3 \\
\hline 50 & 98 & 74.7 & 34.5 & 20.3 & 13.7 & 9.7 & 6.9 & 5.6 & 4.3 & 3.8 \\
\hline 55 & 138 & 81.7 & 41.4 & 24.9 & 15.6 & 10.9 & 8 & 6.3 & 5 & 4.1 \\
\hline 60 & 106 & 85.3 & 41.9 & 26.2 & 16.7 & 11.6 & 8.8 & 6.7 & 5.3 & 4.6 \\
\hline 65 & 137 & 103 & 49.7 & 28.1 & 17 & 11.9 & 9.2 & 7.4 & 5.7 & 4.8 \\
\hline 70 & 187 & 117 & 51.9 & 30.1 & 19.5 & 13.9 & 10.3 & 7.9 & 6.2 & 5.4 \\
\hline 75 & 155 & 123 & 57 & 32.9 & 21.7 & 15.3 & 11.2 & 8.5 & 6.7 & 5.7 \\
\hline 80 & 228 & 138 & 60.9 & 36 & 22.4 & 15.3 & 12.1 & 9.3 & 7.2 & 6 \\
\hline 85 & 233 & 135 & 61.3 & 37.6 & 23.9 & 17 & 12.6 & 9.7 & 7.6 & 6.4 \\
\hline 90 & 246 & 153 & 64.2 & 39.6 & 24.5 & 17.1 & 13.6 & 10.6 & 8.1 & 6.7 \\
\hline 95 & 212 & 155 & 7.6 & 43.7 & 26.6 & 19 & 14.1 & \begin{tabular}{|l|}
10.9 \\
\end{tabular} & 8.5 & 7.1 \\
\hline 100 & 318 & 174 & 72.3 & 46.7 & 28.4 & 20.2 & 14.9 & 11.2 & 9.3 & 7.7 \\
\hline
\end{tabular}

